\title{
CÍRCULO FAMILIAR. FIGURA JURÍDICA LABORAL QUE POSIBILITA EL TRABAJO INFANTIL
}

\author{
Family circle. Legal work figure that enables child labour
}

Juan Pablo SÁNCHEZ TEJEDA ${ }^{*}$

\begin{abstract}
Sumario:
I. Introducción II. Trabajo de menores y trabajo infantil III. Precarización y flexibilización laboral como factores que inciden en la generación de trabajo infantil IV. Círculo familiar y talleres familiares V. Trabajo infantil en el círculo familiar VI. Conclusiones VII. Fuentes de consulta.
\end{abstract}

Resumen: En México está prohibido que las personas menores de quince años trabajen; sin embargo, en la Ley Federal del Trabajo se contempla una figura jurídica que se denomina círculo familiar, con la que se permite que niñas y niños realicen actividades de trabajo, pero sin que se les considere propiamente como trabajadores. Esto provoca la posibilidad de que se genere lo que jurídicamente se considera como trabajo infantil, el cual también está prohibido.

Palabras clave: Círculo familiar, Trabajo infantil, Talleres familiares, Trabajo de menores, Precarización laboral, Flexibilización laboral

Abstract: In Mexico it is forbidden for persons under fifteen years of age to work; however, the Federal Labour Law provides for a legal figure called the family circle, with which girls and boys are allowed to carry out work activities, but without being considered as workers themselves. This causes to the possibility of generating what is legally considered child labor, which is also prohibited.

Keywords: Family circle, child labor, family workshops, child labor, labor precariousness, labor flexibility

\section{Introducción}

En México se encuentra prohibido el trabajo infantil, ya que las personas deben tener quince años, por lo menos, para que puedan ser consideradas legalmente como parte de una relación laboral. Sin embargo, existe una figura en la Ley Federal del Trabajo con la que se permite que personas menores de esa edad puedan realizar actividades de trabajo.

El hecho de que las normas jurídicas permitan que las personas menores de quince años lleven a cabo actividades de trabajo, implica un grave atentado en contra de los niños y niñas, ya que ellos no tienen la responsabilidad u obligación de trabajar para autosatisfacer sus necesidades o para satisfacer las de alguien más.

Ante esta situación, es conveniente explorar y analizar esta contradicción desde la perspectiva jurídica, debido a que las propias normas legales generan la posibilidad de que se produzca el trabajo infantil.

\footnotetext{
* Maestro en Derecho; Profesor Investigador del Departamento de Derecho de la Universidad Autónoma Metropolitana, Unidad Azcapotzalco. 


\section{Trabajo de menores y trabajo infantil}

En materia de derecho laboral, las frases trabajo de menores y trabajo infantil tienen una gran relevancia, debido a que, a través de los años, tanto a nivel nacional (en México) como internacional se ha procurado llevar a cabo la regulación jurídica de la protección de los menores de edad en el ámbito del trabajo. En el caso de México, existen diversos ordenamientos legales que regulan dicha situación; los hay de forma interna en el sistema jurídico mexicano, así como de forma externa, es decir, instrumentos internacionales en los que México es parte. Por antecedentes históricos de dicho país, puede inferirse que si en la actualidad no existieran las normas legales mínimas que regulan el empleo de los menores de edad, sería posible y normal utilizarlos en el ámbito laboral sin observar y respetar sus derechos humanos.

Uno de los elementos más importantes para considerar que el empleo de un individuo se encuentra regulado dentro de lo que se conoce como trabajo de menores, es el factor de la edad física. Pero, entonces, ¿las frases trabajo de menores y trabajo infantil se refieren a lo mismo? Para encontrar algunas posibles semejanzas o diferencias entre estas dos frases, resulta conveniente tomar en cuenta algunos de los ordenamientos jurídicos internos del sistema jurídico mexicano, así como algunos instrumentos internacionales en los que el Estado mexicano es parte.

En cuanto a los ordenamientos internos, en la propia Constitución Política de los Estados Unidos Mexicanos se hace referencia al trabajo de menores. En las fracciones II, III y XI del apartado A del artículo 123 constitucional, se establecen ciertas cuestiones respecto a los menores que se desempeñan como trabajadores. De estas tres fracciones, la que cobra una notable relevancia es la III; lo anterior, debido a que en ella se señala la edad mínima para que un individuo pueda ser considerado como trabajador. La edad mínima que se señala en dicha fracción es la de quince años ya que se establece que está prohibido utilizar a menores de esa edad en el ámbito del trabajo.

Esto es de suma importancia, debido a que esta regla general indica que para que una persona pueda ser considerada como trabajador, debe tener como mínimo quince años de edad física. Dicha fracción no hace distinción alguna entre los sexos masculino y femenino, por lo que debe entenderse que dicho precepto aplica tanto para hombres como para mujeres. De acuerdo con esta fracción, no es lícito emplear a menores de quince años de edad física, debido a que, inclusive, se prohíbe.

En la misma fracción se hace referencia a que aquellos trabajadores que sean mayores de quince años, pero menores de dieciséis, no deberán tener una jornada mayor a seis horas. Con esta disposición, se observa la intención del legislador de brindar un derecho especial para los trabajadores que se encuentren dentro de ese rango de edad.

Asimismo, en las fracciones II y XI del apartado A del artículo 123 constitucional, también se establecen ciertos derechos para los trabajadores que tengan menos de dieciséis años de edad física. Por lo que hace a la fracción II, se señala que se prohíben para estos trabajadores aquellas actividades que sean peligrosas o insalubres, labores después de las diez de la noche, así como trabajo nocturno industrial. Por su parte, en la fracción XI se señala que este tipo de trabajadores no deberán ser utilizados en trabajos de tiempo extraordinario ${ }^{1}$.

Con estas tres fracciones, podemos observar que en la Constitución Federal se establecen derechos de protección laboral para trabajadores que se encuentran dentro del rango de esa edad.

\footnotetext{
${ }^{1}$ En los artículos 50., fracción IV y 178 de la Ley Federal del Trabajo, se prohíbe el trabajo en horas extraordinarias para personas menores de dieciocho años.
} 
Sin embargo, cabe hacer notar que, a pesar de que este tipo de trabajadores no rebasa la edad de dieciséis años, ya son considerados como trabajadores; pero también es conveniente puntualizar que en las tres fracciones se hace referencia a menores, pero no se menciona el término infantil. Por lo que, en la Constitución Política Federal, se conceden derechos especiales, en el ámbito del trabajo, para menores de cierta edad.

Otro de los ordenamientos legales internos que resulta esencial revisar, es la Ley Federal del Trabajo. Los artículos 22 y 22 Bis de esa Ley mantienen una estrecha relación en cuanto al establecimiento de la edad con la que un individuo puede ser considerado como trabajador, así como la prohibición del desempeño en el trabajo cuando el individuo no tenga esa edad mínima. De acuerdo con el primer párrafo del artículo 22, es posible que las personas que tengan quince años, por lo menos, puedan prestar de manera libre sus servicios; sin embargo, para ello deben observarse las diversas limitaciones que se señalan en esa propia Ley. Asimismo, con base en el artículo 22 Bis, se prohíbe que los individuos que tengan menos de quince años puedan ser considerados como trabajadores.

En sincronía con los artículos 22 y 22 Bis, se encuentra la fracción I del artículo 50. de la misma Ley; debido a que, con base en el primer párrafo de este artículo y dicha fracción, se considera que no surte efectos legales la estipulación en la que se señale que los individuos menores de quince años se desempeñen en el ámbito del trabajo. Y no solo eso, el hecho de que, contrario a la ley, se llegase a estipular el trabajo para personas que tengan menos de quince años, esto no impedirá el goce ni el ejercicio de sus derechos. Estos señalamientos aplican, también de acuerdo con el primer párrafo del artículo 50., tanto para la estipulación hecha de manera verbal como para la escrita. Esto es importante debido a que en la propia Ley Federal del Trabajo se prohíbe que personas que tengan menos de quince años, puedan incursionar de manera legal en el ámbito del trabajo.

En este sentido, la propia Ley Federal del Trabajo permite que las personas que se encuentren dentro del rango de edad de quince y menos de dieciocho años, puedan llevar a cabo una relación de trabajo. Los derechos laborales de este tipo de trabajadores se encuentran establecidos en dicha Ley, inclusive, existe un título completo destinado a la regulación de sus derechos, además de diversos artículos del mismo ordenamiento legal que hacen referencia a estos trabajadores.

El Título Quinto Bis, denominado Trabajo de los Menores es aquel en el que, específicamente, se regulan los derechos de este tipo de trabajadores. Comprende del artículo 173 al 180 de dicha Ley. Nótese que la denominación del título hace referencia a trabajo de menores, más no a trabajo infantil. Sin embargo, en el segundo párrafo del artículo 173 se hace mención del trabajo infantil, debido a que se ordena que la Secretaría del Trabajo y Previsión Social, mediante coordinación con autoridades de las entidades federativas en materia laboral, lleve a cabo programas que generen la posibilidad de identificar, así como de erradicar el trabajo infantil.

De lo anterior se desprende que, de acuerdo con la Ley Federal del Trabajo, el trabajo infantil puede ser considerado aquel donde individuos menores de quince años realizan actividades laborales. Esto, debido a que la propia Ley determina que el trabajo infantil debe ser identificado $y$, en consecuencia, erradicado; pero, por otra parte, permite que las personas que tengan como mínimo quince años, ya puedan desempeñarse en el ámbito laboral.

Inclusive, en el propio artículo 362 de esa Ley, se reconoce a aquellos individuos mayores de quince años como trabajadores, además de que se les permite formar parte de sindicatos. Así también, de acuerdo con el párrafo primero del artículo 691 del mismo ordenamiento legal, los menores trabajadores pueden comparecer en un juicio de carácter laboral sin que sea necesario 
que se les otorgue autorización de ningún tipo, debido a que les reconoce la capacidad para ello. Ahora bien, para el caso de que dichos menores no estén asesorados en el juicio, el Tribunal ${ }^{2}$ debe solicitar a la Procuraduría de la Defensa del Trabajo que intervenga con el fin de asesorarles. Asimismo, solo en caso de que los menores de dieciséis años no tengan representante, dicha Procuraduría debe designarles uno.

En consecuencia, al permitírseles ser parte de sindicatos y comparecer en juicios, se demuestra una vez más que los menores trabajadores pueden incursionar en el ámbito laboral y que, por ende, podrían no ser considerados parte del trabajo infantil.

Existe una excepción a esta regla general que se contempla en el artículo 175 Bis, toda vez que diversas actividades que lleven a cabo menores de quince años de edad no serán consideradas como trabajo. Dichas actividades deben estar vinculadas con ámbitos como el deportivo, interpretación artística, desarrollo científico, entre otros; además de que esas actividades deben estar sujetas a las reglas que se establecen en los incisos a, b y c de dicho artículo. Asimismo, esas actividades también deben ser realizadas con la existencia de la responsabilidad, supervisión y cuidado, ya sea de los padres, los tutores o aquellas personas que tengan el ejercicio de la patria potestad.

Esta es una excepción en la que se permite a las personas menores de quince años, realizar ciertas actividades sin que estas se consideren laborales. Pero algo muy importante es que, de acuerdo con el inciso c del referido artículo 175 Bis, los menores de quince años que lleven a cabo dichas actividades sí pueden cobrar contraprestaciones por ellas; incluso, se establece que esas retribuciones, en ningún caso, deberán ser inferiores a las que percibiría como salario un trabajador mayor de quince y menor de dieciocho años de edad. De esta manera, se observa que la ley sí permite que esos menores de quince años desempeñen esas actividades y cobren por ellas algo parecido a la remuneración denominada salario, la cual es propia de los trabajadores. En este sentido, en la ley se determina que esas actividades no deben ser consideradas como trabajo para los menores de esa edad; pero si no existiera tal determinación, se actualizaría, de forma inminente, el supuesto del trabajo infantil.

Dentro de las normas jurídicas internas del sistema jurídico mexicano, podemos utilizar las tesis aisladas y jurisprudenciales. De acuerdo con la tesis aislada emitida por la Primera Sala de la Suprema Corte de Justicia de la Nación con título DISCRIMINACIÓN POR RAZÓN DE EDAD EN EL ÁMBITO LABORAL. SU PROHIBICIÓN NO SE CONTRAPONE A LA EXCLUSIÓN DEL TRABAJO INFANTIL CONTENIDA EN EL ARTÍCULO 123, APARTADO A, FRACCIÓN III, DE LA CONSTITUCIÓN POLÍTICA DE LOS ESTADOS UNIDOS MEXI$\mathrm{CANOS}^{3}$, podemos deducir que trabajo infantil es aquel que realizan las personas menores de quince años.

Con base en esta tesis, el hecho de que la Constitución Federal prohíba la discriminación generada por la edad de los individuos ${ }^{4}$ no implica que a los menores de quince años se les discrimine por no permitírseles laborar y ser considerados como trabajadores; toda vez que si se

\footnotetext{
2 Mientras no inicien operaciones los Tribunales, las Juntas serán quienes sigan conociendo de este tipo de procedimientos. Lo anterior, con base en el artículo Octavo Transitorio del Decreto por el que fueron modificadas diversas disposiciones de la Ley Federal del Trabajo y que fue publicado en el Diario Oficial de la Federación el día 1 de mayo de 2019.

3 Tesis 1a. CDXXXIII/2014 (10a.), Gaceta del Semanario Judicial de la Federación, Décima Época, Libro 13, t. I, diciembre de 2014, p. 230.

4 Esa prohibición de discriminación por edad se establece en el párrafo quinto del artículo 1o. de la Constitución Política de los Estados Unidos Mexicanos.
} 
fija un mínimo de edad para permitirles que puedan incorporarse al mercado laboral es porque se prohíbe el trabajo infantil. Además, vale la pena destacar que en dicha tesis se hace énfasis de que el trabajo infantil produce importantes consecuencias tanto en el acceso a la educación como en la integridad física de los menores de edad.

En cuanto a los instrumentos internacionales en los que México es parte y que tienen relación con estos temas, podemos utilizar el Convenio número 138 de la Organización Internacional del Trabajo, denominado Convenio sobre la edad mínima de admisión al empleo ${ }^{5}$, el cual se encuentra ratificado por México ${ }^{6}$. Es importante tomar en cuenta que, de acuerdo con el artículo 133 de la Constitución Política Federal, los tratados que, entre otras cosas, sean aprobados por el Senado, deben ser considerados Ley Suprema de toda la Unión.

De acuerdo con el párrafo 3, con relación al párrafo 1 del artículo 20. de dicho Convenio sobre la edad mínima de admisión al empleo, para que a una persona se le pueda admitir en algún trabajo o empleo, su edad mínima no debe ser inferior a aquella en la que se concluye la obligación escolar en el país de que se trate, o bien, su edad no debe ser inferior a quince años. Aunque en dicho Convenio se establecen algunas excepciones en ciertas circunstancias, esta es una especie de regla general de edad mínima para que un individuo pueda incursionar en el ámbito laboral. En este sentido, podemos observar que, en sintonía con la Constitución de la República, la Ley Federal del Trabajo y el referido Convenio, en México se determina que las personas deben tener, por lo menos, quince años, para que puedan llevar a cabo actividades consideradas como trabajo.

Algo que vale la pena destacar, es que en el artículo 10. del Convenio sobre la edad mínima, 1973, se dispone que los miembros que tengan vigente dicho Convenio se comprometen a abolir el trabajo de los niños, de manera efectiva, mediante su política nacional. Esto permite inferir, si se toma en cuenta la regla general que se establece en dicho Convenio, que trabajo infantil es aquel que desarrollan los niños y niñas menores de quince años; debido a que se permite el trabajo de los mayores de quince años, pero no el de los menores de esa edad.

Otro instrumento de carácter internacional que tiene relación con estos temas es el Convenio número 182 de la Organización Internacional del Trabajo, denominado Convenio sobre la prohibición de las peores formas de trabajo infantil y la acción inmediata para su eliminación ${ }^{7}$, el cual también se encuentra ratificado por México ${ }^{8}$.

Con base en el artículo 20. de este Convenio sobre las peores formas de trabajo infantil, 1999, el término niño hace referencia a las personas con menos de dieciocho años; pero es importante

\footnotetext{
5 Organización Internacional del Trabajo, C138, Convenio sobre la edad mínima, 1973 (núm. 138); Convenio sobre la edad mínima de admisión al empleo (Entrada en vigor: 19 junio 1976), [en línea], disponible en: https:// www.ilo.org/dyn/normlex/es/f?p=NORMLEXPUB:1210o:o::NO::P1210o_ILO_CODE:C138 (consultada el 21 de marzo de 2019).

6 La fecha de ratificación de dicho Convenio, por parte de México, es 10 de junio de 2015. Véase en OrganizACión Internacional del Trabajo, Ratificación del C138 - Convenio sobre la edad mínima, 1973 (núm. 138), [en línea], disponible en: https://www.ilo.org/dyn/normlex/es/f?p=NORMLEXPUB:1130o:o::NO::P1130o_INSTRUMENT_ID:312283 (consultada el 21 de marzo de 2019).

7 Organización Internacional del Trabajo, C182 - Convenio sobre las peores formas de trabajo infantil, 1999, (núm. 182); Convenio sobre la prohibición de las peores formas de trabajo infantil y la acción inmediata para su eliminación (entrada en vigor: 19 noviembre 200o), [en línea], disponible en: https://www.ilo.org/dyn/normlex/ es/f?p=NORMLEXPUB:1210o:o::NO::P1210o_ILO_CODE:C182 (consultada el 22 de marzo de 2019).

8 La fecha de ratificación de dicho Convenio, por parte de México, es 30 de junio de 20oo. Véase en OrGanizACión Internacional del Trabajo, Ratificación del C182 - Convenio sobre las peores formas de trabajo infantil, 1999, (núm. 182), [en línea], disponible en: https:/www.ilo.org/dyn/normlex/es/f?p=10oo:1130o:0::NO:11300 :P11300_INSTRUMENT_ID:312327 (consultada el 22 de marzo de 2019).
} 
tener en cuenta que dicha determinación respecto a esa edad, es solo para los efectos de este Convenio.

Así pues, si tomamos en cuenta la tesis aislada, los ordenamientos jurídicos internos del sistema jurídico mexicano e instrumentos internacionales que hemos revisado y analizado en este apartado, podemos determinar que en el caso de México las frases trabajo de menores y trabajo infantil no se refieren a lo mismo. Esto, debido a que trabajo de menores es aquel que consiste en la realización de aquellas actividades de carácter laboral permitidas por la ley, que llevan a cabo mujeres u hombres mayores de quince y menores de dieciocho años de edad, y se encuentra regulado en el sistema jurídico mexicano. Por otra parte, trabajo infantil es aquel que consiste en la realización de actividades de carácter laboral que llevan a cabo mujeres u hombres menores de quince años de edad, y se encuentra prohibido en el sistema jurídico mexicano.

\section{Precarización y flexibilización laboral como factores que inciden en la generación de trabajo infantil}

En México, la edad mínima con la que un individuo puede ser considerado trabajador es a partir de los quince años, sin embargo, por diversas razones, menores de esa edad también trabajan. Esto se ha venido recrudeciendo con el paso del tiempo.

A pesar de que en la República mexicana existe la prohibición del trabajo infantil, niños y niñas realizan actividades de carácter laboral; no obstante, esto no es exclusivo del contexto actual, inclusive en el pasado el trabajo de infantil ha sucedido. Un ejemplo de ello es lo que comenta Mario de la Cueva:

En los albores del siglo XIX comprendieron los hombres de buena voluntad que era indispensable prohibir el trabajo de los niños, forma inhumana de obtener beneficios, porque se corría el riesgo de impedir su desarrollo físico y provocar la degeneración de la población, y porque no permitía su concurrencia a las escuelas. Fueron muchas las leyes que se dictaron en ese sentido. La mezquindad del régimen del presidente Díaz se mostró en el célebre laudo arbitral de 7 de enero de 1907, en el que determinó que "no se admitirían los menores de siete años en las fábricas para trabajar", párrafo que es mejor no comentar?.

No es de extrañar que un ejemplo tan lamentable para el caso del trabajo infantil haya sido extraído de la época del régimen del entonces presidente de México, Porfirio Díaz, ya que durante ese periodo gran parte de la población vivió situaciones atroces en el ámbito laboral.

Actualmente, el modo de producción que prevalece en México, como en gran parte del mundo, es el capitalismo. Este modo de producción, en general, ha traído grandes avances científicos y desarrollo tecnológico, pero también ha generado severas consecuencias en el bienestar de las personas, incluso, hasta en el medio ambiente. Aparentemente, el capitalismo ha generado elementos de comodidad y satisfacción para la vida de las personas, sin embargo, también ha provocado que gran cantidad de gente se encuentre inmersa en la pobreza y, en algunos casos, pobreza extrema. Esta situación de afectación no es ajena a México.

El capitalismo actual en México es prácticamente inconcebible si no se toman en cuenta factores como la globalización y el neoliberalismo y, en particular, en el ámbito del trabajo, la precarización y la flexibilización. El empleo de las personas se ha vuelto cada vez más inestable

9 Cunva, Mario de la (2001), El nuevo derecho mexicano del trabajo, t. I: Historia, principios fundamentales, derecho individual y trabajos especiales, $18^{\text {a }}$ ed., México, Porrúa, p. 209. 
e insuficiente para tener una vida digna, por lo que es común que el empleo sirva solamente para subsistir; y eso, cuando los individuos cuentan con empleo formal, ya que también existe el ámbito en el que la gente no tiene ese tipo de empleo y desempeña actividades en el trabajo informal. Alex Munguía Salazar y Claudia G. de la Fuente Morales señalan que: La precarización laboral no es tan sólo un concepto, sino que encierra un conjunto de derechos que no están siendo respetados, resultado de políticas gubernamentales. Sin embargo, dichas políticas han estado enfocadas desde una posición neoliberal bajo un proceso globalizador ${ }^{10}$. Definitivamente, las políticas gubernamentales tienen un rol decisivo en el destino de la población, sobre todo, en el económico.

Con base en el neoliberalismo y la globalización las condiciones en el ámbito del trabajo se han ido modificando, pero, desafortunadamente, en perjuicio de los trabajadores. Eso genera precarización laboral. Los términos, muy mencionados en el argot laboral, productividad y competitividad han sido comprendidos y utilizados, comúnmente, para beneficio de los patrones; no así para beneficio de los trabajadores. Se utiliza el discurso de que, entre mejor le vaya a la empresa mejor les irá a sus trabajadores, pero en la realidad casi nunca es así. Se imponen condiciones de trabajo extenuantes a los empleados y muchas de las veces ellos las toleran por necesidad. Pero al hacer la distribución de la riqueza en las empresas, casi siempre es en beneficio de los patrones y, en consecuencia, resulta inequitativa. En ese sentido, resulta conveniente tomar en cuenta la aseveración de Héctor Santos Azuela y Verónica Santos Méndez:

Dentro de la dinámica moderna nuestro sistema se orienta hacia una política neoliberal cuyos efectos laceran los derechos de los trabajadores, que representan sin duda, el soporte humano productivo del Estado. En esta virtud, con tan dolorosa realidad, las transformaciones a la productividad, las transformaciones a la normatividad positivo-laboral continúan proliferando impunemente, so pretexto de que así lo exigen la globalización y la apertura comercial ${ }^{11}$.

La precarización del trabajo, generalmente, va acompañada de la vulneración de los derechos humanos laborales de los empleados. Cada vez es más común que los trabajadores no reciban o disfruten de aguinaldo, pago de horas extras, pago excedente por los días de descanso obligatorio laborados, vacaciones pagadas, pago de prima vacacional, inscripción a la seguridad social, etcétera. En los casos en que los trabajadores solicitan a los patrones se cumplan sus derechos laborales, la justificación suele ser que no hay dinero para ello; y si cometen la irreverencia de insistir en el cumplimiento de sus derechos, la presunta condena con la que se les tranquilizan sus exacerbados ánimos es la terminación de la relación laboral, o en términos más exactos: el despido.

Situaciones de despido y desempleo provocan que sea cada vez más difícil que los trabajadores tengan estabilidad en el empleo; además, hoy en día existen otros motivos que también ocasionan esa inestabilidad, como son: precarias condiciones laborales, régimen de trabajo (verbigracia, la subcontratación, también conocida como outsourcing), así como la duración de la relación laboral (ejemplos: contrato para tiempo determinado, periodo a prueba y capacitación inicial). Así podemos observar la lamentable y desafortunada flexibilización laboral. Una inte-

\footnotetext{
${ }^{10}$ Munguía Salazar, Alex y Fuente Morales, Claudia G. de la (2017), "Las políticas neoliberales en México y la precariedad laboral", en: Flores SAlgado, Lucerito Ludmila y Munguía SAlazAr, Alex (coords.), México y los costos sociales de las reformas laboral, educativa y energética, México, Benemérita Universidad Autónoma de Puebla, p. 146.

${ }^{11}$ Santos Azuela, Héctor y Santos Méndez, Verónica (2014), Fundamentos y reforma del Derecho del trabajo, México, Porrúa, p. 229.
} 
resante aseveración es la que ofrecen Alex Munguía Salazar y Claudia G. de la Fuente Morales: En la flexibilización del mercado laboral se busca la flexibilización de las relaciones laborales por medio de la reforma de la legislación; en consecuencia, la precarización laboral trae consigo inestabilidad laboral y violación de los derechos laborales ${ }^{12}$. Generalmente, las políticas económicas que permean en el contexto de una sociedad son plasmadas en los ordenamientos jurídicos, ya sea a través de la creación de nuevas leyes, códigos, reglamentos, etcétera, o, incluso, mediante la adición de preceptos o reforma de las normas ya existentes. Un ejemplo de esto es la reforma que se llevó a cabo en el año 2012 a la Ley Federal del Trabajo, la cual, estuvo fuertemente impregnada de ideología neoliberal.

Todos esos factores inmersos en el capitalismo, pero, sobre todo, la precarización y la flexibilización laboral, han provocado que la población, en general, sufra condiciones laborales y de vida muy difíciles y, en algunos casos, hasta crueles. Lo cual, desafortunadamente, genera que los menores de dieciocho años también tengan que llevar a cabo actividades laborales. Alena Garrido Ramón comenta lo siguiente:

El desempleo y el subempleo, la descomposición de la familia, el abandono y otras causas propician que muchos seres humanos se integren desde temprana edad a las relaciones sociales de producción, aunque con frecuencia en condiciones adversas. Por ello los menores de edad desempeñan trabajos domésticos, o laboran en talleres mecánicos, estacionamientos, expendios de gasolina o en las calles o los tianguis, como vendedores de objetos muy variados; si a esto se añade que no siempre cuentan con una persona que se ocupe de su educación, se construye un terreno fértil para la violencia, el alcoholismo, la drogadicción, la prostitución, la delincuencia, las enfermedades, etcétera ${ }^{13}$.

Otra de las razones por las cuales menores de edad tienen que incursionar en el ámbito del trabajo, tal como lo comenta Alena Garrido Ramón, es el abandono. No solo menores que viven con sus padres, familiares, tutores u otras personas desempeñan actividades laborales, sino también aquellos que viven en situación de calle, sin alguien que les brinde atención a sus necesidades.

El hecho de que la situación económica no sea favorable para la población mexicana en general ha ocasionado que personas menores de quince años tengan que incursionar en el ámbito laboral, con lo cual, se produce el trabajo infantil. Esta lamentable situación puede presentarse porque menores de esa edad no tienen a alguien que se encargue de ellos, pero también puede ser generada porque los padres, tutores o terceras personas encargadas de ellos los hacen trabajar.

De esta manera, la precarización y la flexibilización, ambas en lo laboral, además de que tienden a favorecer la pobreza, inciden en la generación de trabajo infantil, debido a que las consecuencias que producen tienen un fuerte impacto negativo en las condiciones económicas y en la vida de la población en general. A causa de eso, menores de quince años, por necesidad o por imposición, tienen que realizar actividades de carácter laboral.

\footnotetext{
${ }^{12}$ Munguía Salazar, Alex y Fuente Morales, Claudia G. de la (2017), op. cit., nota 10, p. 150.

${ }^{13}$ Garrido Ramón, Alena (2017), Derecho individual del trabajo, 2a ed., México, Oxford University Press, colección Textos jurídicos universitarios, p. 179.
} 


\section{Círculo familiar y talleres familiares}

En la Ley Federal del Trabajo se contemplan dos figuras que tienen cierta semejanza por su relación con el ámbito de carácter familiar, sin embargo, se trata de dos supuestos que son distintos. Esas figuras son el círculo familiar y los talleres familiares.

La figura de los talleres familiares aparece contemplada en el Capítulo XV denominado Industria familiar, el cual se encuentra dentro del Título Sexto denominado Trabajos Especiales. De acuerdo con el artículo 351 de dicho ordenamiento legal, en los talleres familiares solo personas específicas llevan a cabo actividades de trabajo; se toma como base a los cónyuges, pero también sus ascendientes, descendientes y pupilos pueden ser considerados parte de ese taller familiar.

De acuerdo con la tesis aislada que tiene por título TALLER FAMILIAR. INTEGRANTES (INTERPRETACIÓN DEL ARTÍCULO 351 DE LA LEY FEDERAL DEL TRABAJO) ${ }^{14}$, cuando se hace referencia a los cónyuges también puede incluirse a los concubinos; cuando se hace referencia a ascendientes, se debe tomar en cuenta solo a los padres, abuelos y bisabuelos de los cónyuges o, en su caso, concubinos; cuando se hace referencia a descendientes, únicamente debe tomarse en cuenta a los hijos, nietos y bisnietos de los cónyuges o concubinos; y cuando se menciona a pupilos, se refiere a ciertos casos específicos de menores de edad o mayores de edad incapacitados sujetos a tutela legítima, también con respecto a los cónyuges o concubinos. Es conveniente destacar que, con base en dicha tesis aislada, la relación que se presenta en el taller familiar es diferente a la que se genera entre trabajador y patrón.

Ahora bien, algo muy importante es que, con base en el artículo 352 de dicha Ley, las disposiciones de este ordenamiento legal no son aplicables a los talleres familiares; no obstante, los únicos supuestos en los que sí les aplica esta Ley es en aquellos concernientes a temas de higiene y seguridad. De esta manera, si se hace una interpretación literal del referido artículo, puede inferirse que en los talleres familiares no se generan relaciones de trabajo, por lo que no hay trabajador o trabajadores vinculados a patrón o patrones; sí hay individuos que desempeñan actividades, pero estas no se llevan a cabo dentro de lo que se concibe como relación de trabajo ${ }^{15}$ en la Ley Federal del Trabajo. Una apreciación interesante es la que ofrecen José Pérez Chávez y Raymundo Fol Olguín: Cabe observar que en muchas ocasiones los talleres familiares se llegan a confundir con las micro y pequeñas empresas; sin embargo, son totalmente diferentes, ya que de acuerdo con la LFT, los talleres familiares son aquellos en los que exclusivamente trabajan los integrantes de una familia, por lo que no cuentan con trabajadores que no sean miembros de ésta ${ }^{16}$. A pesar de que en los talleres familiares hay gente que trabaja, no se configura relación de trabajo alguna; ese es un motivo por el cual las disposiciones de la Ley Federal del Trabajo no les son aplicables.

Por otra parte, en el cuarto párrafo del artículo 23 de la Ley Federal del Trabajo se describe lo que debe entenderse por círculo familiar. En esta figura jurídica se toma como base a los menores de edad. En dicho párrafo se establece: Se entenderá por círculo familiar a los parientes del

\footnotetext{
14 Tesis XIX.10.35 L, Semanario Judicial de la Federación y su Gaceta, Novena Época, t. XXXII, octubre de 2010, p. 3211.

${ }^{15}$ En el primer párrafo del artículo 20 de la Ley Federal del Trabajo se establece lo que debe entenderse por relación de trabajo: Se entiende por relación de trabajo, cualquiera que sea el acto que le dé origen, la prestación de un trabajo personal subordinado a una persona, mediante el pago de un salario.

${ }^{16}$ Pérez Chávez, José y Fol Olguín, Raymundo (2018), Taller de prácticas laborales y de seguridad social, 14a ed., México, Tax Editores Unidos, p. 365.
} 
menor, por consanguinidad, ascendientes o colaterales; hasta el segundo grado. Cuando se menciona parientes por consanguinidad ascendientes hasta el segundo grado, se refiere a padres y abuelos; y cuando se menciona parientes por consanguinidad colaterales hasta el segundo grado, se refiere a hermanos ${ }^{17}$.

La figura de círculo familiar sí se regula por la Ley Federal del Trabajo; vale la pena hacer notar que, incluso, se encuentra contemplada dentro del Capítulo I denominado Disposiciones generales del Título Segundo denominado Relaciones Individuales de Trabajo. De esta manera, puede inferirse que el trabajo realizado por individuos menores de edad está permitido dentro del círculo familiar, inclusive el de los menores de quince años.

\section{Trabajo infantil en el círculo familiar}

Los años de la infancia integran una etapa que trasciende en la personalidad de cualquier ser humano, debido a que tiene que ver con diversas fases de su desarrollo físico e intelectual, así como con diferentes ámbitos en los que se desenvuelve durante toda su vida.

Existen diversos factores que resultan importantes en la infancia de una persona, los cuales, generalmente, deben ser observados y atendidos por sus padres, tutor o quien tenga la obligación de hacerlo con base en la ley, ya que es muy difícil que el propio niño o niña los atiendan por sí mismos. Entre esos factores se encuentran, por ejemplo: comida, vestimenta, educación escolar, vivienda, atención médica, sano esparcimiento, acceso a la cultura. Sin embargo, existen diversas causas por las cuales, en muchos casos, las personas que están encargadas de cuidar y proteger a las niñas y niños no cumplen con su obligación. A pesar de que ese incumplimiento depende de la propia situación y causas particulares de cada caso en concreto, pueden existir causas generales que inciden en él o, incluso, lo provocan; verbigracia: pobreza, distribución inequitativa de la riqueza, bajos salarios, desempleo, políticas de gobierno que no benefician a la mayoría de la población, precarización y flexibilización laboral, corrupción, entre otras.

Pero, independientemente de las causas particulares o generales que incidan o provoquen el incumplimiento del cuidado y protección de las niñas y niños, estos no deben asumir la responsabilidad de autosatisfacer sus propias necesidades ni, mucho menos, deben trabajar para satisfacerlas.

Podría generarse la idea de que, ante un estado de necesidad o pobreza, los niños y niñas deben trabajar para ayudar a sus padres, tutor o a la persona que con base en la ley tiene la obligación de su protección y cuidado, pero esa forma de proceder ¿estará debidamente justificada? ¿Será una idea socialmente correcta? ¿Será una decisión familiar apropiada? ¿Legalmente se permite tal situación? Es posible que las respuestas a estas interrogantes sean diferentes debido al punto de vista, vivencias y experiencias de cada persona; pero en cuanto a la interrogante de carácter legal, es posible analizar una figura jurídica que, probablemente, permite que el trabajo

\footnotetext{
17 Cfr. Chávez Castillo, Raúl (2011), Derecho de familia y sucesorio (Curso Derecho Civil IV), 2 ${ }^{\mathrm{a}}$ ed., México, Porrúa, pp. 5 y 7; así como, Muñoz Rocha, Carlos I. (2015), Derecho familiar, México, Oxford University Press, colección Textos jurídicos universitarios, pp. 194, 196, 197, 198 y 202. Además, artículos 292 al 300 del Código Civil Federal; así como, artículos 292 al 300 del Código Civil para el Distrito Federal (actualmente también denominado: Código Civil para la Ciudad de México).
} 
infantil pueda llevarse a cabo en México. Esa figura, la cual se encuentra regulada en la Ley Federal del Trabajo, es el círculo familiar ${ }^{18}$.

De acuerdo con esta figura, los niños y niñas pueden trabajar sin que sus actividades sean consideradas como parte de una relación de trabajo ${ }^{19}$. En el primer párrafo del artículo 23 de la Ley Federal del Trabajo se determina lo siguiente: Cuando las autoridades del trabajo detecten trabajando a un menor de quince años fuera del círculo familiar, ordenará que de inmediato cese en sus labores. Al patrón que incurra en esta conducta se le sancionará con la pena establecida en el artículo 995 Bis de esta Ley. Con base en este párrafo, no se permite que los menores de quince años trabajen fuera de su círculo familiar, por lo que, si se hace una interpretación a contrario sensu, puede inferirse que la ley sí permite que los menores de quince años de edad puedan trabajar dentro del círculo familiar.

Tan es así, que el quinto párrafo del artículo 23 de la Ley Federal del Trabajo establece: Cuando los menores de dieciocho años realicen alguna actividad productiva de autoconsumo, bajo la dirección de integrantes de su círculo familiar o tutores, éstos tendrán la obligación de respetar y proteger los derechos humanos de los menores y brindar el apoyo y las facilidades necesarias para que los mismos concluyan, por lo menos, su educación básica obligatoria. Con este párrafo se confirma la posibilidad que concede la ley para que las personas menores de quince años puedan trabajar en su círculo familiar. Si bien en este párrafo no se hace referencia específica a los menores de quince años, sí se hace referencia, de manera general, a todos los menores de dieciocho.

El hecho de que en el referido párrafo quinto se mencione la frase: realicen alguna actividad productiva de autoconsumo, no implica que los menores de edad no lleven a cabo actividades de trabajo; es decir, formalmente no están empleados, pero sí están realizando trabajo. En el segundo párrafo del artículo 8o. de la Ley Federal del Trabajo se señala lo que debe entenderse por trabajo: Para los efectos de esta disposición, se entiende por trabajo toda actividad humana, intelectual o material, independientemente del grado de preparación técnica requerido por cada profesión u oficio. Si únicamente se toma en cuenta este párrafo segundo, sin concatenarlo con algún otro precepto legal, puede observarse que el trabajo puede llevarse a cabo sin necesidad de estar contratado por alguien para realizarlo; además de que la preparación técnica para desempeñar un trabajo no es factor determinante para llevarlo a cabo. Evidentemente, existe un sinfín de actividades de trabajo que requieren conocimientos previos para que puedan ser desempeñadas, pero esto se debe, entre otros motivos, a que se pretende que haya un resultado idóneo y no se generen daños o perjuicios innecesarios.

La Suprema Corte de Justicia de la Nación señala que: El trabajo es una tarea que no necesariamente da al trabajador una retribución económica. El empleo, por el cual puede entenderse "acción de emplear" y "ocupación, oficio", es una posición o cargo que un individuo ocupa en una empresa o institución, donde su trabajo (físico o intelectual) es debidamente remunerado ${ }^{20}$. En consecuencia, si las personas menores de quince años de edad llevan a cabo dentro de su círculo familiar alguna actividad productiva de autoconsumo (como se menciona en la Ley), aunque no estén contratadas para desempeñar un trabajo y, por ende, no se les pague salario por ello, puede considerarse que, de todas formas, realizan un trabajo. En este sentido, si se toma en cuenta

\footnotetext{
${ }^{18}$ Véase el apartado IV. CÍRCULO FAMILIAR Y TALLERES FAMILIARES del presente artículo de investigación.

19 Véase lo que de acuerdo con la Ley Federal del Trabajo debe entenderse por Relación de trabajo, nota 15.

${ }^{20}$ Suprema Corte de Justicia de la Nación (2017), Derecho del trabajo: parte general, México, Suprema Corte de Justicia de la Nación, p. 27.
} 
lo que debe entenderse por relación de trabajo ${ }^{21}$, de acuerdo con la Ley Federal del Trabajo, entre el menor de quince años de edad y los integrantes de su círculo familiar no se configura un vínculo jurídico laboral; sin embargo, sí desempeña un trabajo.

Generalmente, las personas que integran su círculo familiar y que los ponen a trabajar no les consultan para saber si están o no de acuerdo con tal imposición. Se le sujeta a un ritmo de vida, en muchos de los casos, totalmente desgastante, máxime si aparte de trabajar cursan su educación escolar. Existen las ideas de que cuando se pone a trabajar al menor de edad se le hace un bien para su futura adultez, que se le enseña a ser responsable, que se le prepara para mantener y sacar adelante al negocio, que se le educa para obtener el fruto de su trabajo con su propio esfuerzo, que se le inculca ganarse el pan con el sudor de su frente; pero, en realidad ¿esos serán los motivos principales? O quizá solo son pretextos y justificaciones para hacer que las personas menores de quince años trabajen en el círculo familiar.

Definitivamente, inculcar en los menores de edad valores morales como la responsabilidad, honradez y respeto por el trabajo es de beneficio para ellos y necesario para mejorar la sociedad mexicana; sin embargo, esos valores no deben servir de pretexto o justificación para hacer que trabajen y, mucho menos, los menores de quince años.

Las personas menores de edad deben disfrutar de lo que se denomina jurídicamente como alimentos $^{22}$. De acuerdo con Raúl Chávez Castillo: El derecho de alimentos se ha definido como la facultad jurídica que tiene una persona denominada acreedor alimentista para exigir a otra llamada deudor alimentario lo necesario para vivir, derivada de la relación que se tenga con motivo del parentesco consanguíneo o civil, del matrimonio, del divorcio y, en determinados casos, del concubinato ${ }^{23}$. Por su parte, Carlos I. Muñoz Rocha define el derecho de alimentos de la manera siguiente: La facultad jurídica de interés público que tiene un acreedor para exigir a un deudor, en virtud de la relación jurídica familiar, lo necesario para ayudar a su subsistencia, en los términos y parámetros que fija la ley ${ }^{24}$. Disfrutar de los alimentos es un derecho humano que tienen las niñas y niños en México, el cual se encuentra consagrado en el párrafo noveno del artículo 4o. de la Constitución Política de los Estados Unidos Mexicanos; además, con base en el décimo párrafo del mismo artículo, tanto los ascendientes como los tutores y los custodios de las niñas y niños, tienen la obligación de preservar y exigir el cumplimiento de ese derecho humano a los alimentos.

Es importante destacar que, a pesar de que el círculo familiar de los menores de edad abarca a sus padres, abuelos y hermanos, el quinto párrafo del artículo 23 de la Ley Federal del Trabajo contempla también a sus tutores; además de que, en ese quinto párrafo, se determina que tanto los integrantes de su círculo familiar como sus tutores están obligados a proteger y respetar los derechos humanos de los menores. Por lo cual, si se toman en cuenta de manera conjunta estos preceptos expuestos de la Constitución Política Federal y de la Ley Federal del Trabajo, se puede observar que las niñas y niños no deben trabajar para satisfacer sus propias necesidades refe-

\footnotetext{
${ }^{21}$ Véase lo que de acuerdo con la Ley Federal del Trabajo debe entenderse por Relación de trabajo, nota 15.

${ }^{22}$ En el artículo 308 de Código Civil Federal se establece: Los alimentos comprenden la comida, el vestido, la habitación y la asistencia en casos de enfermedad. Respecto de los menores los alimentos comprenden, además, los gastos necesarios para la educación primaria del alimentista, y para proporcionarle algún oficio, arte o profesión honestos y adecuados a su sexo y circunstancias personales. Así también, en el artículo 314 del mismo ordenamiento legal se determina: La obligación de dar alimentos no comprende la de proveer de capital a los hijos para ejercer el oficio, arte o profesión a que se hubieren dedicado.

${ }^{23}$ Chávez Castillo, Raúl (2011), op. cit., nota 17, p. 16.

${ }^{24}$ Muñoz Rocha, Carlos I. (2015), op. cit., nota 17, p. 213. 
rentes al derecho humano de alimentos. Los niños y niñas son quienes tienen esa prerrogativa, más no esa obligación.

Este es un asunto que atañe a toda la sociedad mexicana ${ }^{25}$ y no se debe circunscribir solo al interior de las familias o, en su caso, de los círculos familiares, porque en la medida en que el trabajo infantil se oculte, disfrace, tolere, minimice o consienta seguirá existiendo y aumentando. Una destacada aseveración es la que hace Carlos I. Muñoz Rocha:

Sin duda en México tenemos una gran deuda con la sociedad, con las familias, pero sobre todo tenemos una deuda de proporciones descomunales con la niñez, porque todos somos cómplices de los males que la aquejan, de su ¿educación?, de su desnutrición, de su miseria económica, de su abandono notorio, de su explotación sexual, del tráfico de infantes, etc. Les hemos robado su inocencia, les hemos privado de sus mejores años; como decía Miguel de Unamuno, tenemos un letrero en la frente que dice: iEres reo de muerte! ${ }^{26}$

Con la figura jurídica círculo familiar se permite que los niños y las niñas realicen actividades de trabajo, pero sin considerarlos ni denominarlos trabajadores ${ }^{27}$. Esta figura es una trampa legal ${ }^{28}$ debido a que con ella se pretende hacer creer que las niñas y niños solo realizan actividades productivas con finalidad de autoconsumo y que, por ende, no se encuentran inmersos y activos dentro del ámbito laboral. Sin embargo, independientemente del nombre o denominación que se le dé a esa figura jurídica, su objetivo es permitir que las niñas y niños trabajen sin que esto genere una consecuencia legal negativa para quienes los hacen trabajar. Esto representa un punto a favor del neoliberalismo, es decir, más mano de obra y, además, gratuita.

Ahora bien, las niñas y niños que verdaderamente realizan actividades productivas con fines de autoconsumo lo hacen para autosatisfacer sus propias necesidades, pero eso también es trabajo infantil. Cómo es posible que en el primer párrafo del artículo 23 de la Ley Federal del Trabajo se ordene sancionar ${ }^{29}$ a los patrones que utilicen a menores de quince años en actividades de trabajo y, de manera implícita, se permita trabajar a los menores de esa edad en el círculo familiar. Es una incongruencia que en la Ley Federal del Trabajo se prohíba el trabajo infantil y, al mismo tiempo, se permita que niñas y niños trabajen en su círculo familiar.

\section{Conclusiones}

Las niñas y niños deben disfrutar su etapa infantil. Ellos deben dedicar su tiempo a actividades acordes a su edad. Su madurez física e intelectual en esa etapa es insuficiente para que se dediquen a realizar actividades de trabajo. Ellos deben llevar a cabo actividades que les permitan

${ }^{25}$ En el caso de la Ciudad de México, en el artículo 940 del Código de Procedimientos Civiles para el Distrito Federal (actualmente también denominado Código de Procedimientos Civiles para la Ciudad de México) se establece: Todos los problemas inherentes a la familia se consideran de orden público, por constituir aquélla la base de la integración de la sociedad.

${ }^{26}$ MuÑoz Rocha, Carlos I. (2015), op. cit., nota 17, p. 230.

${ }^{27}$ En el primer párrafo del artículo 8o. de la Ley Federal del Trabajo se establece que: Trabajador es la persona física que presta a otra, física o moral, un trabajo personal subordinado.

${ }^{28}$ De acuerdo con el Diccionario de la lengua española de la Real Academia Española, trampa legal significa: 1. f. Acto ilícito que se cubre con apariencias de legalidad. [en línea], disponible en: https://dle.rae.es/?id=aGlB829 (consultada el 13 de abril de 2019).

${ }^{29}$ La sanción es la establecida en el artículo 995 Bis de la Ley Federal del Trabajo, que a la letra dice: Al patrón que infrinja lo dispuesto en el artículo 23, primer párrafo de esta Ley, se le castigará con prisión de 1 a 4 años y multa de 250 a 5000 veces la Unidad de Medida y Actualización. 
fortalecer su sano desarrollo; deben realizar, principalmente, actividades lúdicas, culturales, deportivas y, por supuesto, escolares.

Delegarles una responsabilidad que no les corresponde es totalmente injusto. Hacerlos trabajar para que ellos mismos sufraguen sus gastos o satisfagan sus propias necesidades es lamentable, sobre todo porque ellos, en esa etapa, aún son indefensos y, en muchos de los casos, no están en las condiciones de poder contradecir las decisiones de las personas que los obligan a trabajar. Hacerlos que trabajen, aun en el círculo familiar, con la idea de que colaboren económicamente para cubrir los gastos de su familia, es atentar en contra de los propios niños y niñas.

Quizá para algunos sectores de la sociedad mexicana resulte más fácil poner a trabajar a las niñas y niños que enfrentar los problemas que perjudican gravemente la situación económica de la población general de este país, tales como: pobreza, pobreza extrema, inequitativa distribución de la riqueza, corrupción, desempleo, bajos salarios, precarización y flexibilización laboral, entre otros.

Es conveniente que esta situación que afecta a las niñas y niños se revise y analice en diversos foros, espacios y ámbitos y, en su oportunidad, se valore la posibilidad de reformar la Ley Federal del Trabajo para eliminar la figura jurídica denominada círculo familiar.

Hoy nos toca a los adultos cuidar y proteger a las personas que actualmente son niñas y niños, ellos algún día crecerán y también tendrán la obligación de hacerlo con los futuros niños y niñas.

\section{Fuentes de consulta}

\section{Bibliografía}

Chávez Castillo, Raúl (2011), Derecho de familia y sucesorio (Curso Derecho Civil IV), 2ª ed., México, Porrúa.

Cueva, Mario de la (2001), El nuevo derecho mexicano del trabajo, t. I: Historia, principios fundamentales, derecho individual y trabajos especiales, $18^{\text {a }}$ ed., México, Porrúa.

Garrido Ramón, Alena (2017), Derecho individual del trabajo, 2ª ed., México, Oxford University Press, colección Textos jurídicos universitarios.

Lóyzaga De la Cueva, Octavio (2017), El derecho del trabajo. Un análisis crítico, $2^{\mathrm{a}}$ ed., México, Universidad Autónoma Metropolitana, Unidad Azcapotzalco, colección Derecho.

Munguía Salazar, Alex y Fuente Morales, Claudia G. de la (2017), "Las políticas neoliberales en México y la precariedad laboral”, en: Flores SAlgado, Lucerito Ludmila y Munguía Salazar, Alex (coords.), México y los costos sociales de las reformas laboral, educativa y energética, México, Benemérita Universidad Autónoma de Puebla.

Muñoz Rocha, Carlos I. (2015), Derecho familiar, México, Oxford University Press, colección Textos jurídicos universitarios.

Pérez Chávez, José y Fol Olguín, Raymundo (2018), Taller de prácticas laborales y de seguridad social, $14^{\mathrm{a}}$ ed., México, Tax Editores Unidos. 
Reynoso Castillo, Carlos (2015), Los derechos humanos laborales, México D.F., Universidad Autónoma Metropolitana-Tirant Lo Blanch.

Santos Azuela, Héctor y Santos Méndez, Verónica (2014), Fundamentos y reforma del Derecho del trabajo, México, Porrúa.

Soberanes Díez, José María y Soberanes Fernández, José Luis (2016), Los derechos humanos del orden jurídico mexicano en la Constitución Política de México y en los tratados internacionales: sus antecedentes y evolución, México, Miguel Ángel Porrúa.

Suprema Corte de Justicia de la Nación (2017), Derecho del trabajo: parte general, México, Suprema Corte de Justicia de la Nación.

\section{Legislación}

C138 - Convenio sobre la edad mínima, 1973 (núm. 138); Convenio sobre la edad mínima de admisión al empleo (Entrada en vigor: 19 junio 1976).

C182 - Convenio sobre las peores formas de trabajo infantil, 1999 (núm. 182); Convenio sobre la prohibición de las peores formas de trabajo infantil y la acción inmediata para su eliminación (Entrada en vigor: 19 noviembre 2000).

Código Civil Federal.

Código Civil para el Distrito Federal (Código Civil para la Ciudad de México).

Código de Procedimientos Civiles para el Distrito Federal (Código de Procedimientos Civiles para la Ciudad de México).

Constitución Política de los Estados Unidos Mexicanos.

Ley Federal del Trabajo.

Ley Federal del Trabajo. Instrumentos internacionales de la OIT sobre trabajo de menores, Salarios mínimos vigentes (2019), México, Gallardo Ediciones.

\section{Páginas electrónicas}

Asamblea Legislativa del Distrito Federal. Códigos, [en línea], disponible en: http://www.aldf. gob.mx/codigos-107-4.html

Cámara de Diputados. H. Congreso de la Unión. Leyes federales vigentes, [en línea], disponible en: http://www.diputados.gob.mx/LeyesBiblio/index.htm

Diccionario de la lengua española. Real Academia Española, [en línea], disponible en: https:// dle.rae.es/?id=DgIqVCc

Organización Internacional del Trabajo, [en línea], disponible en: https://www.ilo.org/global/ lang--es/index.htm 
Ciencia Jurídica, ISSN impresa: 2007-3577, ISSN electrónica: 2007-6142 https://cienciajuridica.ugto.mx DOI: https://doi.org/10.15174/cj.v9i17.325

Suprema Corte de Justicia de la Nación. Semanario Judicial de la Federación, [en línea], disponible en: https://sjf.scjn.gob.mx/sjfsist/Paginas/tesis.aspx 